\title{
SPEKTRALEIGENSCHAFTEN DES DIRAC-OPERATORS- \\ DIE FUNDAMENTALLÖSUNG SEINER \\ WÄRMELEITUNGSGLEICHUNG UND \\ DIE ASYMPTOTENENTWICKLUNG DER ZETA-FUNKTION
}

\section{H. DLUBEK \& TH. FRIEDRICH}

\section{Einleitung}

Besitzt eine geschlossene, orientierte Riemannsche Mannigfaltigkeit $X^{n}$ eine Spin-Struktur, so wird im assoziierten Spinorbündel $\delta$ durch kovariante Ableitung und Clifford-Multiplikation ein elliptischer Differentialoperator D 1. Ordnung, der sogenannte Dirac-Operator, definiert. Die Eigenwerte $\lambda_{i}$ sowie die Dimensionen $m_{i}$ der Eigenunterräume des Operators $D^{2}$ bestimmen seine Zeta-Funktion

$$
\zeta(t)=\sum_{i=0}^{\infty} m_{i} \cdot e^{-\lambda_{l} t}
$$

deren asymptotisches Verhalten an der Stelle $t=0$ in der vorliegenden Arbeit untersucht wird. Zu diesem Ziel geben wir eine Konstruktion der Fundamentallösung $E(x, y, t)$ der Wärmeleitungsgleichung

$$
\left(\frac{\partial}{\partial t}+D^{2}\right) u=0
$$

an, welche die Geometrie des Spinorbündels, insbesondere die in ihm induzierte kovariante Ableitung und Parallelverschiebung benutzt und erhalten:

Theorem. Sei $X^{n}(n \geqslant 3)$ eine geschlossene Spin-Mannigfaltigkeit und $D$ der im Spinorbündel wirkende Dirac-Operator. Die Zeta-Funktion $\zeta(t)$ des Operators $D^{2}$ besitzt an der Stelle $t=0$ die asymptotische Entwicklung

$$
\zeta(t) \sim(4 \pi t)^{-n / 2} \sum_{j=0}^{\infty} d_{j} t^{j}
$$

Received, November 7, 1977, and, in revised form, March 31, 1978. 
mit

$$
\begin{aligned}
& d_{0}=\operatorname{dim} \delta \cdot \operatorname{Vol}(X), \\
& d_{1}=-\frac{\operatorname{dim} \delta}{12} \int_{X^{n}} R(x) d x, \\
& d_{2}=\frac{\operatorname{dim} \delta}{4320} \int_{X^{n}}\left(15 R(x)^{2}-21\|\Re(x)\|^{2}-24 \rho(x)^{2}\right) d x .
\end{aligned}
$$

Danach wenden wir uns der Frage zu, welche Größen einer Riemannschen Mannigfaltigkeit mit Spin-Struktur durch die Spektren des Laplace-Operators $\Delta$ und des Operators $D^{2}$ zusammen determiniert werden. Neben Resultaten über gewisse Klassen 4-dimensionaler Mannigfaltigkeit, in denen die Eulersche Charakteristik, die Signatur bzw. das arithmetische Geschlecht durch diese Spektren bestimmt werden, erhalten wir Anwendungen auf $n$-dimensionale Räume konstanter Schnittkrümmung. Insbesondere sind die Sphäre $S^{n}$ und der reell-projektive $\operatorname{Raum} \mathbf{P}^{4 k+3}(\mathbf{R})$ in der Klasse aller Spin-Mannigfaltigkeit durch die Spektren von $\Delta$ und $D^{2}$ bis auf Isometrie festgelegt.

\section{Die Fundamentallösung und die Zeta-Funktion eines elliptischen Operators}

Sei $X^{n}$ eine glatte, kompakte, $n$-dimensionale Mannigfaltigkeit ohne Rand, und $E$ ein komplexes Vektorbündel mit hermitischer Metrik über ihr. Den Raum der glatten Schnitte dieses Vektorbündels bezeichnen wir mit $\Gamma(E)$. Die Metrik in $E$ gestattet es, mit jedem Differentialoperator $P: \Gamma(E) \rightarrow \Gamma(E)$ den zu ihm adjungierten $P^{*}: \Gamma(E) \rightarrow \Gamma(E)$ zu betrachten, welcher durch die Gleichung

$$
\int_{X}\left(P s, s^{\prime}\right)=\int_{X}\left(s, P^{*} s^{\prime}\right), \quad s, s^{\prime} \in \Gamma(E),
$$

eindeutig definiert wird. Ist $P$ elliptisch der Ordnung $m$, so untersucht man das Cauchy-Problem der zum Operator $P$ gehörigen Wärmeleitungsgleichung ( WLG)

$$
\begin{aligned}
\frac{\partial u(x, t)}{\partial t}+P P^{*} u(x, t) & =0, \\
u(x, 0) & =f(x),
\end{aligned}
$$

für $t \geqslant 0, f \in L^{2}(E)$ und $u(\cdot, t) \in \Gamma(E)$.

Es ist wohlbekannt (vgl. [1], [12]), daß im Bündel $E \bowtie E$ über $X \times X$ ein Schnitt $E(x, y, t)(t>0)$ existiert-die sogenannte Fundamentallösung des 
Cauchy-Problems der $W L G-$, welche durch die nachstehenden drei Eigenschaften eindeutig charakterisiert ist:

1. $E$ ist einmal in $t$ und $(2 m)-m a l$ in $x$ und $y$ differenzierbar.

2. $(\partial / \partial t) E(x, y, t)+\left(P P^{*}\right)_{x} E(x, y, t)=0$.

3. Für alle $s \in \Gamma(E)$ und alle $x \in X$ gilt

$$
s(x)=\lim _{t \rightarrow 0} \int_{X} E(x, y, t) \circ s(y) d y .
$$

Die Spur der Fundamentallösung

$$
\zeta(t)=\int_{X} \operatorname{Tr} E(x, x, t) d x
$$

ist die Zeta-Funktion des Operators $P P^{*}$, die sich bekanntlich(vgl. [1], [12]) durch die Eigenwerte $0=\lambda_{0}<\lambda_{1}<\lambda_{2}<\cdots \rightarrow \infty$ und die Dimensionen $m_{0}, m_{1}, \cdots$ der entsprechenden Eigenunterräume dieses Operators durch die Formel

$$
\zeta(t)=\sum_{i=0}^{\infty} m_{i} e^{-\lambda_{i} t}
$$

ausdrücken läßt.

Von Interesse ist das asymptotische Verhalten dieser Funktion an der Stelle $t=0$. Nach Seeley (vgl. [1], [12]) ist das asymptotische Verhalten der Funktion $\zeta(t)$ für $t \rightarrow 0$ durch

$$
\zeta(t) \underset{t \rightarrow 0^{+}}{\sim} \frac{1}{t^{n / 2 m}} \cdot \sum_{k=0}^{\infty} a_{k}\left(P P^{*}\right) t^{k}
$$

mit den Koeffizeinten $a_{k}\left(P P^{*}\right)=\int_{X} \mu_{k}$ gegeben, wobei die Maße $\mu_{k}$ durch eine lokale Konstruktion aus dem Operator $P$ erhalten werden können.

Diese Koeffizienten wurden für eine Reihe spezieller Operatoren bereits berechnet (vgl. [2], [8], [11] im Fall $P P^{*}=$ Laplace-operator einer Riemannschen Mannigfaltigkeit auf Funktionen, sowie [5] für den Laplaceoperator auf $p$-Formen; siehe auch [6]). Da wir für den Dirac-Operator $D$ eine geometrische Konstruktion der Fundamentallösung angeben und daraus die ersten Koeffizienten der Asymptotenentwicklung der zu $D^{2}$ gehörigen ZetaFunktion erhalten wollen, beschreiben wir an dieser Stelle die Konstruktion der Fundamentallösung etwas näher. Wir beschränken uns mit Hinblick auf diese Anwendung auf Operatoren $P$ der Ordnung 1 . Zwei von einem Parameter $t \geqslant 0$ stetig abhängende Schnitte $A(x, y, t), B(x, y, t)$ in $E \otimes E$ über $X \times X$ kann man nach der Formel

$$
(A * B)(x, y, t)=\int_{0}^{t} \int_{X} A(x, u, t-\vartheta) \circ B(u, y, \vartheta) d u d \vartheta
$$


falten und erhält somit wiederum einen vom Parameter $t>0$ stetig abhängenden Schnitt $(A * B)(x, y, t)$ in dem gleichen Bündel. Wenden wir diese Operation mehrfach auf ein und denselben Schnitt $A$ an, so schreiben wir $A * \cdots * A=A^{* i}$.

Gegeben sei nun eine ganze Zahl $k>n / 2+2$. Unter einer Parametrix verstehen wir einen Schnitt $W(x, y, t)(t>0)$ in $E \bowtie E$ über $X \times X$ mit folgenden drei Eigenschaften:

1. $\left(\partial / \partial t+P P^{*}\right) W(x, y, t)$ läßt sich auf $t=0$ stetig fortsetzen.

2. $s(x)=\lim _{t \rightarrow 0} \int_{X} W(x, y, t) \circ s(y) d y, s \in \Gamma(E)$.

3. Es existiert ein $T>0$ und eine Konstante $c$, so daß für alle $t \in[0, T]$ gilt

$$
\left\|\left(\frac{\partial}{\partial t}+P P^{*}\right) W(x, y, t)\right\| \leqslant c t^{k-n / 2} .
$$

Lemma 1. Ist $W$ eine Parametrix, so gilt:

1. Die Reihe $Q=\sum_{i=1}^{\infty}(-1)^{i+1}\left(\left(\partial / \partial t+P P^{*}\right) W\right)^{* i}$ ist konvergent und definiert einen vom Parameter $t \geqslant 0$ stetig abhängenden Schnitt in $E \otimes E$.

2. $E:=W-W * Q$ ist die Fundamentallösung der WLG zum Operator $P$.

3. Für kleine $t$ gilt:

$$
\|E(x, y, t)-W(x, L y, t)\| \leqslant c^{\prime} t^{k-n / 2+1} .
$$

Beweis. Der Beweis entspricht dem in [2] für den Laplace-Operator auf Funktionen gegebenen. Den Operator $\partial / \partial t+P P^{*}$ bezeichnen wir mit $Z$ und beweisen zuerst, daß $Q=\sum_{i=1}^{\infty}(-1)^{i+1}(Z W)^{* i}$ gleichmäßig konvergiert. Aus der dritten Eigenschaft der Parametrix ergibt sich $\|Z W(x, y, t)\|<C=$ $c T^{k-n / 2}$ für alle $x, y \in X, t \in[o, T]$. Ist $V$ das Volumen von $X$, so ergibt eine mehrfache Anwendung der Faltungsformel die Abschätzung $(0<t \leqslant T)$

$$
\left\|(Z W)^{* i}(x, y, t)\right\| \leqslant \frac{C^{i} V^{i-1} t^{i-1}}{(i-1) !} \leqslant \frac{C^{i} V^{i-1} T^{i-1}}{(i-1) !} .
$$

Damit hat $Q$ die Majorante

$$
\sum_{i=1}^{\infty} \frac{C^{i} V^{i-1} T^{i-1}}{(i-1) !}=C \cdot e^{C V T}
$$

und konvergiert gleichmäßig gegen einen stetigen Schnitt. Eine direkte Rechnung zeigt (vg. [2]), daß die Differenz $W-W * Q$ die charakteristischen Eigenschaften der Fundamentallösung erfüllt und somit ergibt sich $E=W-$ $W * Q$. Wir zeigen nun die dritte in Lemma 1 behauptete Eigenschaft. Aus $\|Z W(x, y, t)\| \leqslant c \cdot t^{k-n / 2}$ folgt wie oben die Abschätzung

$$
\|Q(x, y, t)\| \leqslant C_{1} t^{k-n / 2}
$$


welche zeigt, daß der Schnitt $R(x, y, t)=Q(x, y, t) / t^{k-n / 2}$ um $t=0$ beschränkt ist. Es ist

$$
(W * Q)(x, y, t)=\int_{0}^{t} \vartheta^{k-n / 2} \int_{X} W(x, u, t-\vartheta) \circ R(u, y, \vartheta) d u d \vartheta
$$

Die zweite Eigenschaft der Parametrix liefert

$$
\lim _{\vartheta \rightarrow t} \int_{X} W(x, u, t-\vartheta) \circ R(u, y, \vartheta) d u=R(x, y, t)
$$

und sichert somit die Beschränktheit von

$$
\int_{X} W(x, u, t-\vartheta) \circ R(u, y, \vartheta) d u \text { für } 0 \leqslant \vartheta \leqslant t .
$$

Daraus erhält man unmittelbar die Behauptung.

Folgerung 1. $\zeta(t)=\int_{X} \operatorname{Tr} W(x, x, t)+o\left(t^{k-n / 2+1}\right)$.

\section{Der Dirac-Operator}

In diesem Abschnitt stellen wir einige bekannte Tatsachen über den DiracOperator zusammen (vg. [4], [7], [14]). Gegeben sei eine parakompakte, orientierte Riemannsche Mannigfaltigkeit $X^{n}$ der Dimension $n \geqslant 3$ und bezeichne $\left(P, \pi, X^{n} ; S O(n)\right)$ das assoziierte $S O(n)$-Hauptfaserbündel. Eine Spin-Struktur ist ein Hauptfaserbündel $\left(Q, \pi, X^{n} ; \operatorname{Spin}(n)\right)$ und eine überlagerung $\lambda: Q \rightarrow P$ derart, daß

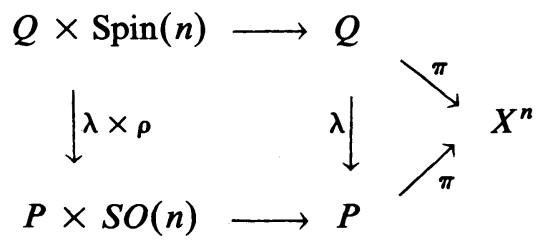

kommutiert. Hierbei ist $\rho: \operatorname{Spin}(n) \rightarrow S O(n)$ die universelle Überlagerung der speziellen orthogonalen Gruppe. Ist $X^{n}$ kompakt, so existiert bekanntlicherweise genau dann eine Spin-Struktur, falls die zweite Stiefel-WhitneyKlasse von $X^{n}$ verschwindet. In diesem Fall werden die Spin-Strukturen durch $H^{1}\left(X^{n}, \mathbf{Z}_{2}\right)$ klassifiziert (vg. [9]).

Für unsere weiteren Überlegungen fixieren wir eine Spin-Struktur. Sind $s o(n)$ bzw. $\operatorname{spin}(n)$ die Lie-Algebren der entsprechenden Gruppen, so hebt

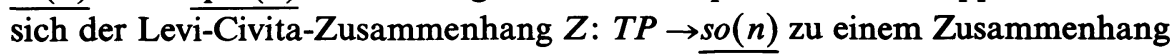


$\hat{Z}: T Q \rightarrow \underline{\operatorname{spin}(n)}$ derart, daß

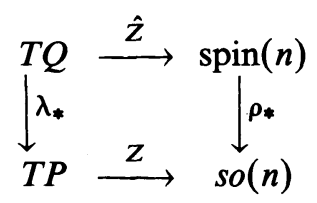

ein kommutatives Diagramm ist. Im zur Spin-Darstellung $\Delta_{n}$ der Gruppe $\operatorname{Spin}(n)$ assoziierten Spinorbündel $\delta=Q \times_{\operatorname{Spin}(n)} \Delta_{n}$ induziert der Zusammenhang $\hat{Z}$ eine kovariante Ableitung

$$
\nabla^{\S}: \Gamma(\delta) \rightarrow \Gamma\left(T^{*}\left(X^{n}\right) \otimes \delta\right)
$$

sowie eine Parallelverschiebung

$$
\tau_{\gamma}: \delta_{\gamma(0)} \rightarrow \delta_{\gamma(1)}
$$

entland einer Kurve $\gamma$ in $X$.

Sei $s=\left(s_{1}, \cdots, s_{n}\right): V \rightarrow P$ ein lokales Reper von orthonormierten Vektorfeldern über einer einfach-zusammenhängenden, offenen Menge V. Der Zusammenhang $Z: T P \rightarrow s o(n)$ induziert die lokale Zusammenhangsform $Z^{s}=Z \circ s_{*}: T V \rightarrow \underline{s o(n)}$. Wählen wir in $s o(n)$ die Standardbasis $E_{i j}(i<j)$, so erhalten wir durch

$$
Z^{s}=\sum_{i<j} \omega_{i j} E_{i j}
$$

die lokalen Formen $\omega_{i j}(i<j)$, welche wir durch $\omega_{i j}+\omega_{j i}=0$ für alle Indizes $(i, j)$ definieren können. Nach [10] kann man $\underline{\operatorname{spin}(n)}$ mit der linearen Hülle der Produkte $e_{i} e_{j}(i<j)$ in der Cliffordalgebra $\operatorname{Cliff}\left(\mathbf{R}^{n}\right)$ identifizieren $\left(e_{1}, \cdots, e_{n}\right.$ ist eine orthonormierte Basis des $\left.\mathbf{R}^{n}\right)$ und dabei wird das Differential $\rho_{*}: \underline{\operatorname{spin}(n) \rightarrow \operatorname{so}(n)}$ durch $\rho_{*}\left(e_{i} e_{j}\right)=2 E_{i j}$ beschrieben. Heben wir den Schnitt $s$ zu einem Schnitt $\hat{s}: V \rightarrow Q$, so können wir wiederum die lokale Zusammenhangsform $\hat{Z}^{\hat{s}}=\hat{Z} \circ \hat{s}_{*}: T V \rightarrow \underline{\operatorname{spin}(n)}$ betrachten und erhalten

$$
\hat{Z}^{\hat{s}}=\sum_{i<j} \frac{1}{2} \omega_{i j} e_{i} e_{j} \text {. }
$$

Ist nun $u_{\varepsilon}$ eine orthonormierte Basis des Spin-Moduls $\Delta_{n}$, so erhalten wir durch $\eta_{\varepsilon}(x)=\left[\hat{s}(x), u_{\varepsilon}\right]$ orthonormierte Schnitte $\eta_{\varepsilon}$ in $\delta$ über $V$. Die Kovariante Ableitung $\nabla^{\delta}$ wird dann durch

$$
\nabla_{t}^{\delta} \eta_{e}=\sum_{l<m} \frac{1}{2} \omega_{l m}(t)\left(s_{l} s_{m}\right) \cdot \eta_{e}
$$

beschrieben (vgl. [13, Seite 151]), wobei $\left(s_{l} s_{m}\right) \cdot \eta_{\varepsilon}=\left[\hat{s}(x), e_{l} e_{m} u_{\varepsilon}\right]$ die Clifford-Multiplikation des Spinors $\eta_{\varepsilon}$ mit den Vektoren $s_{l}$ und $s_{m}$ bezeichnet.

Die Riemannsche Metrik gestattet es, $T^{*}\left(X^{n}\right)$ mit $T\left(X^{n}\right)$ zu identifizieren. 
Weiterhin induziert die Clifford-Multiplikation einen Homomorphismus $\mu$ : $T\left(X^{n}\right) \otimes \mathcal{S} \rightarrow \mathcal{\delta}$. Der Dirac-Operator $D$ wird nun als Superposition folgender Abbildungen definiert:

$$
\Gamma(\delta) \stackrel{\nabla^{\delta}}{\rightarrow} \Gamma\left(T^{*}\left(X^{n}\right) \otimes \delta\right) \approx \Gamma\left(T\left(X^{n}\right) \otimes \delta\right) \stackrel{\mu}{\rightarrow} \Gamma(\delta) .
$$

$\mathrm{D}$ ist ein formal selbstadjungierter, elliptischer Differentialoperator 1. Ordnung. Ist $\left(s_{1}, \cdots, s_{n}\right)$ ein orthonormietes Reper von Vektorfeldern auf einer offenen Menge $U \subset X^{n}$ und $u \in \Gamma(\delta)$ ein Schnitt im Spinorbündel, so gilt

$$
D(u)=\sum_{j=1}^{n} s_{j} \cdot \nabla_{s_{j}}^{\delta}(u) .
$$

Ist $R$ die Skalarkrümmung des Riemannschen Raumes $X^{n}$, dann ergibt sich folgende Formel (vgl. [7]) für das Quadrat $D^{2}$ des Dirac-Operators

$$
D^{2}(u)=\frac{1}{4} R u-\sum_{j=1}^{n} \nabla_{s_{j}}^{\delta} \nabla_{s_{j}}^{\delta} u-\sum_{j=1}^{n} \operatorname{div}\left(s_{j}\right) \nabla_{s_{j}}^{\delta} u \text {. }
$$

\section{Die Konstruktion der Fundamentallösung der zum}

\section{Dirac-Operator $D$ gehörigen Wärmeleitungsgleichung}

In diesem Abschnitt der Arbeit konstruieren wir eine Folge von Parametrixen $W_{k}$, welche gemäß Folgerung 1 das asymptotische Verhalten der Zeta-Funktion des Operators $D^{2}=D D^{*}$ an der Stelle $t=0$ beschreiben.

Weil $X$ kompakt ist, existiert eine positive Zahl $\varepsilon>0$ so, daß sich jedes Punktepaar $(x, y)$ der Menge

$$
U_{\varepsilon}=\{(x, y) \in X \times X \mid r(x, y)<\varepsilon\}
$$

deren Abstand $r(x, y)$ kleiner als $\varepsilon$ ist, durch eine eindeutig bestimmte minimale Geodätische $\gamma$ verbinden läßt. Mittels des Exponentials $\exp _{y}$ : $T_{y} X \rightarrow X$ führen wir Normalkoordinaten um den Punkt $y$ ein und definieren auf der Menge $U_{e}$ eine Funktion $\theta=\theta(x, y)$ durch

$$
\theta(x, y)=\sqrt{\operatorname{det}\left(g_{i j}(x)\right)},
$$

wobei $g_{i j}$ die Koeffizienten der Metrik in diesem Koordinatensystem sind. Unter der Anwendung eines Differentialoperators auf einen Schnitt oder eine Funktion, welche über $X \times X$ definiert sind, verstehen wir im weiteren immer dessen Anwendung auf die erste Variable.

Lemma 2. Sei $\Psi \in \Gamma(\delta 凶 \mathcal{S})$ ein Schnitt im Bündel $\delta \bowtie \delta$ über $X \times X$ und $f: X \times X \rightarrow \mathrm{C}$ eine glatte Funktion, welche nur vom Quadrat des geodätischen 
Abstandes $r^{2}=r^{2}(x, y)$ abhängt, $f=f\left(r^{2}\right)$. Dann gilt über $U_{e}: D^{2}(f \Psi)(x, y)=$ $\left(D^{2} \Psi\right) f-\left(4 r^{2} f^{\prime \prime}+2 f^{\prime}\left(r\left(\theta^{\prime} / \theta\right)+n\right)\right) \Psi-4 f^{\prime} \nabla_{\dot{\gamma}(1)}^{\delta} \Psi$, wobei $\gamma$ die minimale Geodätische von $y$ nach $x$ und $\dot{\gamma}$ ihre Ableitung ist.

Beweis. $\Delta$ bezeichne den Laplace-Operator der Riemannschen Mannigfaltigkeit. Aus der im Abschnitt 3 zitierten Formel für $D^{2}$ ergibt sich

$$
D^{2}(f \Psi)=f D^{2} \Psi+\Delta(f) \Psi-2 \nabla_{\mathrm{grad} f}^{\delta} \Psi .
$$

Der Laplace-Operator einer nur von $r$ abhängigen Funktion $h=h(r): U_{e} \rightarrow$ C drückt sich durch

$$
\Delta(h)=-h^{\prime \prime}(r)-h^{\prime}(r)\left(\frac{\theta^{\prime}}{\theta}+\frac{n-1}{r}\right)
$$

aus (vgl. [2]). Wenden wir diese Formel auf $f=f\left(r^{2}\right)$ an, so erhalten wir

$$
\Delta(f)=-4 r^{2} f^{\prime \prime}\left(r^{2}\right)-2 f^{\prime}\left(r^{2}\right)\left(r \frac{\theta^{\prime}}{\theta}+n\right) \text {. }
$$

Andererseits ergibt sich für den Gradienten von $f$-indem wir in den Normalkoordinaten $x_{1}, \cdots, x_{n}$ um $y$ rechnen und $g^{i j} x_{j}=x_{i}$ sowie $\dot{\gamma}(1)=x_{i} \partial / \partial x_{i}$ benutzen

$$
\begin{aligned}
\operatorname{grad} f & =\sum_{i, j} g^{i j} \frac{\partial f\left(r^{2}\right)}{\partial x_{j}} \frac{\partial}{\partial x_{i}}=\sum_{i, j} g^{i j} f^{\prime}\left(r^{2}\right) \frac{\partial r^{2}}{\partial x_{j}} \frac{\partial}{\partial x_{i}} \\
& =\sum_{i, j} g^{i j} f^{\prime}\left(r^{2}\right) 2 x_{i} \frac{\partial}{\partial x_{i}}=\sum_{i} f^{\prime}\left(r^{2}\right) 2 x_{i} \frac{\partial}{\partial x_{i}} \\
& =2 f^{\prime}\left(r^{2}\right) \dot{\gamma}(1) .
\end{aligned}
$$

Setzen wir in der Formel für $D^{2}(f \Psi)$ jetzt die Ausdrücke für $\Delta(f)$ und grad $f$ ein, so erhalten wir die Behauptung. q.e.d.

Für die Konstruktion der Parametrix $W_{k}$ benötigen wir einen Schnitt $I$ in

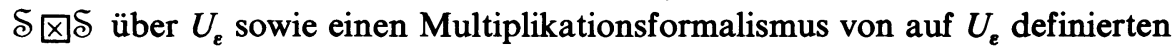
Schnitten in diesem Bündel. Sind $p_{1}, p_{2}$ die Projektionen von $X \times X$ auf die erste und zweite Achse, so identifizieren wir $\delta \bigotimes \mathcal{S} \operatorname{mit} \operatorname{Hom}\left(p_{2}^{*}(\mathcal{S}), p_{1}^{*}(\mathcal{S})\right)$. Einem Schnitt $\Phi \in \Gamma(\mathcal{\bigotimes} \mathcal{S})$ entspricht bei dieser Identifizierung eine Familie von Homomorphismen $\Phi(x, y): \delta_{y} \rightarrow \delta_{x}$. Den Schnitt $I \in \Gamma\left(\delta 区 \delta / U_{e}\right)$ erhalten wir nun dadurch, daß wir jedem Punktepaar $(x, y) \in U_{\varepsilon}$ die Parallelverschiebung im Bündel $\mathcal{S}$ entlang der von diesen beiden Punkten eindeutig bestimmten Geodätischen $\gamma$ zuordnen:

$$
I(x, y)=\tau_{\gamma}: \delta_{y} \rightarrow \delta_{x} .
$$

Ist also $\left\{e_{i}(y)\right\}$ eine orthonormierte Basis von $\delta_{y}$ und $\left\{e_{i}(x)\right\}$ deren Parallelverschiebung entlang $\gamma$, so gilt

$$
I(x, y)=\sum_{i} e_{i}(x) \otimes e_{i}(y)
$$


Sind $\Phi$ und $\Psi$ zwei Schnitte in $\delta \bowtie \delta$ über der Menge $U_{e}$, so haben wir gemäß der angegebenen Identifikation Homomorphismen $\Phi(x, y): \delta_{y} \rightarrow \delta_{x}$ und $\Psi(x, y): \delta_{y} \rightarrow \delta_{x}$. Durch

$$
(\Phi \circ \Psi)(x, y)=\Phi(x, y) I(x, y)^{-1} \Psi(x, y)
$$

wird ein Homomorphismus $(\Phi \circ \Psi)(x, y): \delta_{y} \rightarrow \delta_{x}$, also ein Schnitt $\Phi \circ \Psi$

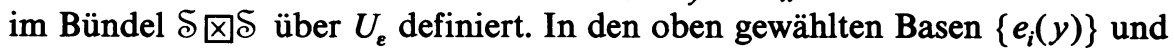
$\left\{e_{i}(x)\right\}$ gilt dann mit

$$
\begin{aligned}
& \Phi(x, y)=\sum_{i, j} \varphi^{i j}(x, y) e_{i}(x) \otimes e_{j}(y), \\
& \Psi(x, y)=\sum_{i, j} \psi^{i j}(x, y) e_{i}(x) \otimes e_{j}(y)
\end{aligned}
$$

die Formel

$$
(\Phi \circ \Psi)(x, y)=\sum_{i, j, k} \varphi^{i k}(x, y) \psi^{k j}(x, y) e_{i}(x) \otimes e_{j}(y) .
$$

Über $U_{\varepsilon}$ definierte Schnitte kann man nach Vektorfeldern differenzieren. Ist nämlich $t$ ein auf $V$ definiertes Vektorfeld und $\Psi$ ein Schnitt in $\delta \bigotimes \mathcal{S}$ über $U_{e}$, so wird durch

$$
\begin{aligned}
\Psi(x, y) & =\sum_{i, j} \psi^{i j}(x, y) e_{i}(x) \otimes e_{j}(y), \\
t(\Psi)(x, y) & =\sum_{i, j} t\left(\psi^{i j}(x, y)\right) e_{i}(x) \otimes e_{j}(y)
\end{aligned}
$$

ein Schnitt $t(\Psi)$ im $\ 凶 \widehat{S}$ über $U_{\varepsilon} \cap(V \times X)$ definiert. Dann gilt

$$
\nabla_{t}^{\delta}(\Phi \circ \Psi)=\Phi \circ t(\Psi)+\left(\nabla_{t}^{\delta} \Phi\right) \circ \Psi .
$$

Aus dieser Formel und Lemma 2 erhalten wir mittels

$$
\begin{aligned}
\nabla_{\dot{\gamma}(1)}^{\delta} \Psi & =\nabla_{\dot{\gamma}(1)}^{\delta}(I \circ \Psi)=I \circ(\dot{\gamma}(1) \Psi)+\left(\nabla_{\dot{\gamma}(1)}^{\delta} I\right) \circ \Psi \\
& =r \cdot I \circ \frac{\partial}{\partial r} \Psi+0=r \cdot \frac{\partial}{\partial r} \Psi
\end{aligned}
$$

das folgende

Lemma 3. Seien $f$ eine auf $U_{\varepsilon}$ definierte, nur vom Quadrat des geodätischen Abstandes $r$ abhängige Funktion und $\Psi$ ein über $U_{\varepsilon}$ definierter Schnitt in $\delta$ $వ$. Dann gilt $D^{2}(f \Psi)=D^{2}(\Psi) f-\left(4 r^{2} f^{\prime \prime}+2 f^{\prime}\left(r\left(\theta^{\prime} / \theta\right)+n\right)\right) \Psi-4 r f^{\prime}(\partial / \partial r) \Psi$.

Folgerung 2. Die Funktion $f$ sei auf $U_{\varepsilon}$ definiert durch

$$
f(x, y)=f\left(r^{2}\right)=(4 \pi t)^{-n / 2} e^{-r^{2}(x, y) / 4 t} \text {. }
$$


Dann gilt

$$
D^{2}(f \Psi)=f D^{2}(\Psi)-\frac{\partial f}{\partial t} \Psi+\frac{r}{2 t} \frac{\theta^{\prime}}{\theta} f \Psi+\frac{r}{t} f \frac{\partial}{\partial r} \Psi
$$

Zur Konstruktion der gesuchten Parametrix $W_{k}$ betrachten wir zuerst einen Schnitt $H_{k}$ über $U_{e}$

$$
H_{k}=\sum_{j=0}^{k} f U_{j} t^{j}
$$

und bestimmen die Schnitte $U_{j}$ so, daß

$$
\left(D^{2}+\frac{\partial}{\partial t}\right) H_{k}=f D^{2}\left(U_{k}\right) t^{k}
$$

gilt. Danach erhalten wir $W_{k}$ aus $H_{k}$, indem wir $H_{k}$ nach vorheriger Glättung außerhalb von $U_{\varepsilon}$ Null legen. $Z u$ diesem Ziel berechnen wir unter Verwendung von Folgerung $2\left(D^{2}+\partial / \partial t\right) H_{k}$ und bekommen

$$
\begin{aligned}
\left(D^{2}+\partial / \partial t\right) H_{k}= & f \sum_{j=0}^{k}\left[D^{2}\left(U_{j-1}\right)+\left(j+\frac{1}{2} r \theta^{\prime} / \theta+r(\partial / \partial r)\right) U_{j}\right] t^{j-1} \\
& +f D^{2}\left(U_{k}\right) t^{k} .
\end{aligned}
$$

Die Bedingungen, welche wir an die Schnitte $U_{j}$ stellen müssen, lauten demnach:

$$
\begin{aligned}
\left(\frac{r}{2} \frac{\theta^{\prime}}{\theta}+r \frac{\partial}{\partial r}\right) U_{0} & =0 \\
D^{2}\left(U_{j-1}\right)+\left(j+\frac{r}{2} \frac{\theta^{\prime}}{\theta}+r \frac{\partial}{\partial r}\right) U_{j} & =0, \quad j=1, \cdots, k .
\end{aligned}
$$

Die Lösungen dieser Differentialgleichungen ergeben folgende Rekursionsformel für die Schnitte $U_{j}$ (vgl. [2]):

$$
\begin{aligned}
& U_{0}(x, y)=\theta^{-1 / 2}(x, y) I(x, y), \\
& U_{j}(x, y)=-\theta^{-1 / 2}(x, y) \int_{0}^{1} \theta^{1 / 2}\left(z_{\xi^{\prime}} y\right) \xi^{j-1} \tau_{\gamma\left(z_{\xi}, x\right)}\left[D^{2}\left(U_{j-1}\right)\left(z_{\xi^{\prime}} y\right)\right] d \xi,
\end{aligned}
$$

wobei $z_{\xi}=\exp _{y}\left(\xi \exp _{V_{y}}^{-1}(x)\right)$, und $\tau_{\gamma\left(z_{\xi}, x\right)}$ die bereits eingeführte Parallelverschiebung $\tau$ im Spinorbündel $\mathcal{S}$ von $z_{\xi}$ nach $x$ ist.

Sei nun $\alpha: X \times X \rightarrow[0,1]$ eine glatte Funktion, die nur vom Quadrat des geodätischen Abstandes abhängt und folgende beiden Eigenschaften erfüllt:

$$
\alpha(x, y)= \begin{cases}1 & \text { falls } r(x, y) \leqslant \varepsilon / 4 \\ 0 & \text { falls } r(x, y) \geqslant \varepsilon / 2\end{cases}
$$


Satz 1. Für $k>n / 2+2$ ist $W_{k}=\alpha H_{k}$ eine Parametrix.

Beweis. $\mathrm{Zu}$ jedem $1>0$ existiert eine Konstante $C_{1}$ so, daß für alle $\varepsilon / 4 \leqslant r \leqslant \varepsilon / 2$ und $0 \leqslant t \leqslant T$

$$
|f(t)|=\left|(4 \pi t)^{-n / 2} e^{-r^{2} / 4 t}\right| \leqslant C_{1} t^{1}
$$

gilt. Die erste Eingenschaft, welche an eine Parametrix gestellt wird, ergibt sich für $W_{k}$ daraus, daß dieser Schnitt im Bereich $r(x, y)>\varepsilon / 4$ auf $t=0$ fortsetzbar ist und für $r(x, y) \leqslant \varepsilon / 4$

$$
\begin{aligned}
\left(D^{2}+\frac{\partial}{\partial t}\right) W_{k} & =\left(D^{2}+\frac{\partial}{\partial t}\right) H_{k}=f \cdot D^{2}\left(U_{k}\right) t^{k} \\
& =(4 \pi)^{-n / 2} e^{-r^{2} / 4 t} t^{k-n / 2} D^{2}\left(U_{k}\right)
\end{aligned}
$$

gilt. Die Abschätzung $\left\|\left(D^{2}+\partial / \partial t\right) W_{k}\right\| \leqslant c t^{k-n / 2}$ erhalten wir, indem wir die linke Seite jeweils über $U_{\varepsilon / 4}$ und $U_{\varepsilon / 2}-U_{\varepsilon / 4}$ entsprechend unter Verwendung von Lemma 3 und der eingangs erwähnten Eigenschaft (1) der Funktion $f$ wie folgt behandeln:

$$
\begin{aligned}
\left\|\left(D^{2}+\frac{\partial}{\partial t}\right) W_{k}\right\|= & \left\|\left(D^{2}+\frac{\partial}{\partial t}\right) H_{k}\right\| \leqslant(4 \pi)^{-n / 2} \cdot 1 \cdot \max _{U_{e} / 4}\left\|D^{2}\left(U_{k}\right)\right\| \cdot t^{k-n / 2}, \\
\left\|\left(D^{2}+\frac{\partial}{\partial t}\right) W_{k}\right\|= & \left\|D^{2}\left(\alpha H_{k}\right)+\alpha \frac{\partial}{\partial t} H_{k}\right\| \\
\leqslant & \left\|\alpha\left(D^{2}+\frac{\partial}{\partial t}\right) H_{k}\right\|+\left|4 r^{2} \alpha^{\prime \prime}+2 \alpha^{\prime}\left(r \frac{\partial^{\prime}}{\theta}+n\right)\right|\left\|H_{k}\right\| \\
& +\left|4 \alpha^{\prime} r\right|\left\|\frac{\partial}{\partial r} H_{k}\right\| \\
& \leqslant C_{1} t^{k-n / 2}+C_{2} t^{k-n / 2}+C_{3} t^{k-n / 2} \\
& \leqslant C t^{k-n / 2} .
\end{aligned}
$$

Wir berechnen nun den Grenzwert

$$
\lim _{t \rightarrow 0} \int_{X} W_{k}(x, y, t) \circ s(x) d y,
$$

indem wir das Integral in die Summen

$$
\int_{X}=\int_{\{y \in X / r(x, y)<\varepsilon / 4\}}+\int_{\{y \in X / r(x, y)>\varepsilon / 4\}}
$$

zerlegen. Aus (1) folgt dann unmittelbar, daß der Grenzwert des zweiten Integrals gleich Null ist. Das vergleibende Integral transformieren wir mittels 
der Exponentialabbildung $\exp _{x}: T_{x} X \rightarrow X$ und erhalten

$$
\begin{aligned}
\lim _{t \rightarrow 0} \int_{X} W_{k}(x, y, t) & \circ s(y) d y \\
= & \lim _{t \rightarrow 0} \int_{\{y \in X / r(x, y)<\varepsilon / 4\}} W_{k}(x, y, t) \cdot s(y) d y \\
= & \lim _{t \rightarrow 0} \sum_{j=0}^{k} t^{k} \int_{\{y \in X / r(x, y)<\varepsilon / 4\}} f(x, y, t) U_{j}(x, y) \circ s(y) d y \\
= & \lim _{t \rightarrow 0} \sum_{j=0}^{k} t^{j} \int_{T_{x} X} f\left(x, \exp _{x} z, t\right) \cdot U_{j}\left(x, \exp _{x} z\right) s\left(\exp _{x} z\right) \\
& \cdot \theta\left(x, \exp _{x} z\right) d z .
\end{aligned}
$$

A us $\lim _{t \rightarrow 0} \int_{\mathbf{R}^{n}}(4 \pi t)^{-n / 2} e^{-|x-z|^{2} / 4 t} g(z) d z=g(x)$ folgt nun $\lim _{t \rightarrow 0} \int_{X} W_{k}(x, y, t) \circ s(y) d y=U_{0}(x, x) \circ s(x) \cdot \theta(x, x)=s(x)$. q.e.d.

Mit Folgerung 1 ergibt sich für die Zeta-Funktion des Operators $D^{2}$.

Folgerung 3. $\zeta(t) \underset{t \rightarrow 0^{+}}{\sim}(4 \pi t)^{-n / 2} \sum_{j=0}^{\infty}\left(\int_{X} \operatorname{Tr} U_{j}(x, x) d x\right) t^{j}$.

Bezeichnung. $\quad d_{j}=\int_{X} \operatorname{Tr} U_{j}(x, x) d x$.

Satz 2. Die Spuren $\operatorname{Tr} U_{j}(y, y)$ sind universelle, $O(n)$-invariante Polynome im Krümmungstensor $R=\left(R_{i j k l}\right)$ des Levi-Civita-Zusammenhanges auf $X$ und seinen Ableitungen $D^{k} \mathcal{R}$. Der Grad $x_{k}$, mit dem $D^{k} \Re$ in einem Monom des die Spur $\operatorname{Tr} U_{j}$ bestimmenden Polynoms $P_{j}^{n}$ auftreten kann, wird durch

$$
j=\sum_{k}\left(1+\frac{k}{2}\right) x_{k}
$$

gegeben.

Beweis. Wir beweisen induktiv, daß $f_{j}(x, y)=\operatorname{Tr} \tau_{\gamma(x, y)} U_{j}(x, y)$ in einer Normalenumgebung von y bezüglich $x$ eine Taylorentwicklung besitzt, deren Koeffizienten universelle Polynome in $\Re(y), D^{1} \Re(y), \cdots$ sind. Weil sich die Koeffizienten der Metrik in der Form

$$
g_{i j}(x)=\delta_{i j}+\frac{1}{3} R_{i k j l} x^{k} x^{l}+\cdots
$$

darstellen lassen (vg. [1]), besitzt

$$
f_{0}(x, y)=\operatorname{dim} \mathcal{S}\left(\operatorname{det}\left(g_{i j}(x)\right)\right)^{-1 / 4}
$$

eine derartige Entwicklung. Aus der Rekursionsformel für $U_{j}$ ergibt sich

$$
f_{j}(x, y)=-\theta^{-1 / 2}(x, y) \int_{0}^{1} \theta^{1 / 2}\left(z_{\xi}, y\right) \xi^{j-1} \operatorname{Tr} \tau_{\gamma\left(z_{\xi}\right)}\left(D^{2} U_{j-1}\left(z_{\xi}, y\right)\right) d \xi .
$$

Benutzen wir nun die leicht einzusehende Formel

$$
t\left(\operatorname{Tr} \tau_{\gamma(x, y)} W(x, y)\right)=\operatorname{Tr} \tau_{\gamma(x, y)}(t W(x, y)),
$$


in welcher $t$ ein Vektor aus $T_{x}(X)$ ist, sowie

so erhalten wir

$$
D^{2}=\frac{1}{4} R-\sum_{i} \nabla_{s_{i}}^{\delta} \nabla_{s_{i}}^{\delta}-\sum_{i} \operatorname{div}\left(s_{i}\right) \nabla_{s_{i}}^{\S}
$$

$$
\begin{aligned}
f_{j}(x, y)= & -\theta^{-1 / 2}(x, y) \int_{0}^{1} \theta^{1 / 2}\left(z_{\xi}, y\right) \xi^{j-1}\left\{\frac{1}{4} R\left(z_{\xi}\right) f_{j-1}\left(z_{\xi}, y\right)+\Delta f_{j-1}\left(z_{\xi}, y\right)\right. \\
& -\operatorname{Tr} \tau_{\gamma\left(z_{\xi}, y\right)}\left(\sum_{i} \nabla_{s_{i}}^{\delta} \nabla_{s_{i}}^{\delta} I+\operatorname{div}\left(s_{i}\right) \nabla_{s_{i}}^{\delta} I\right) \cdot f_{j-1}\left(z_{\xi}, y\right) \\
& \left.-2 \sum_{i} \operatorname{Tr} \tau_{\gamma\left(z_{\xi}, y\right)}\left(\nabla_{s_{i}}^{\delta} I\right) \cdot s_{i}\left(f_{j-1}\right)\left(z_{\xi}, y\right)\right\} d \xi .
\end{aligned}
$$

Aus $\nabla_{s_{i}}^{\delta} e_{\varepsilon}=\Sigma_{e^{\prime}} \Gamma_{i, \varepsilon}^{e^{\prime}} e_{\varepsilon^{\prime}}$ sowie aus der Tatsache, daß $\Gamma_{i, \varepsilon}^{e^{\prime}}$ Linear-Kombinationen der Zusammenhangskoeffizienten $\omega_{i j}\left(s_{k}\right)$ des Levi-Civita-Zusammenhanges sind und der Definition des Schnittes $I$ ergibt sich nun, daß

$$
\operatorname{Tr} \tau_{\gamma\left(z_{\xi}, y\right)}\left(\nabla_{s_{i}}^{\delta} \nabla_{s_{i}}^{\delta} I+\operatorname{div}\left(s_{i} \nabla_{s_{i}}^{\delta} I\right)\right), \quad \operatorname{Tr} \tau_{\gamma\left(z_{\xi}, y\right)}\left(\nabla_{s_{i}}^{\delta} I\right)
$$

Taylorentwicklungen der gesuchten Art besitzen. Induktiv erhalten wir daraus die Taylorentwicklung von $f_{j}(x, y)$ und wegen $\operatorname{Tr} U_{j}(y, y)=f_{j}(y, y)$ die erste Behauptung des Satzes. Die $O(n)$-Invarianz der Polynome erhält man wie in [2].

Die Änderung des Dirac-Operators unter konformer Deformation der Metrik läßt sich durch eine geeignete Identifikation der Spinorbündel durch $\left(D^{2}\right)^{a g}=a^{-1}\left(D^{2}\right)^{g}$ beschreiben (vgl. [7]). Dieser Zusammenhang garantiert, daß sich der in [2] gegebene Beweis der Gleichung $j=\Sigma_{k}(1+k / 2) x_{k}$ auf unseren Fall überträgt. q.e.d.

Nach dem Satz von H. Weyl über die Invarianten der orthogonalen Gruppe erhalten wir aus Satz 2

$$
\begin{aligned}
& \operatorname{Tr} U_{1}(y, y)=k(n) R(y), \\
& \operatorname{Tr} U_{2}(y, y)=\alpha(n) R(y)^{2}+\beta(n)\|\Re(y)\|^{2}+\gamma(n) \rho(y)^{2}+\delta(n) \Delta R(y),
\end{aligned}
$$

wobei $R=R_{j i i j}$ die Skalarkrümmung, $\|\Re\|=\sqrt{R_{i j k l}^{2}}$ die Länge des Krümmungstensors und $\rho=\sqrt{\sum_{i, k}\left(\sum_{i} R_{j i i k}\right)^{2}}$ die Länge des Ricci-Tensors ist.

5. Berechnung der ersten Koeffizienten $d_{0}, d_{1}, d_{2}$ in der

Asymptotenentwicklung der Zeta-Funktion von $D^{2}$

Wir wenden uns jetzt der Berechnung der ersten Koeffizienten $d_{j}=$ $\int_{X^{n}} \operatorname{Tr} U_{j}(x, x) d x \mathrm{zu}$, welche in der Asymptotenentwicklung

$$
\zeta(t) \underset{t \rightarrow 0^{+}}{\sim}(4 \pi t)^{-n / 2} \sum_{j=0}^{\infty} d_{j} t^{j}
$$


der Zeta-Funktion $\tau(t)$ des Operators $D^{2}$ auftreten. Aus $U_{0}(x, x)=$ $\theta^{-1 / 2}(x, x) I(x, x)=I(x, x)$ folgt unmittelbar

$$
d_{0}=\operatorname{dim} \mathcal{S} \cdot \operatorname{Vol}(X) \text {. }
$$

Über die Koeffizienten $d_{1}$ und $d_{2}$ wissen wir bereits, daß diese die Form

$$
\begin{aligned}
& d_{1}=k(n) \int_{X^{n}} R(x) d x, \\
& d_{2}=\int_{X^{n}}\left(\alpha(n) R(x)^{2}+\beta(n)\|\Re(x)\|^{2}+\gamma(n) \rho(x)^{2}\right) d x
\end{aligned}
$$

haben, wobei $k(n), \alpha(n), \beta(n), \gamma(n)$ nur von der Dimension der Mannigfaltigkeit abhängende Größen sind. Um diese zu bestimmen, können wir daher eine Serie von Test-Mannigfaltigkeit einsetzen-welche die Sphären $\boldsymbol{S}^{\boldsymbol{n}}$ sein werden-und für sie $d_{1}$ und $d_{2}$ direkt berechnen. Deshalb wenden wir uns der Untersuchung des Quadrates des Dirac-Operators $D^{2}$ und des im Abschnitt 4 konstruierten Schnittes $I$ auf der Sphäre $S^{n}$ zu.

Satz 3. Sei $S^{n}$ die Standardsphäre und $\left(s_{1}, \cdots, s_{n}\right)$ ein orthonormiertes Reper von Vektorfeldern, welches durch Parallelverschiebung aus einem Punkt $y \in S^{n}$ entsteht. Dann gilt in einer Umgebung von $y$

1. $\Sigma_{i} \nabla_{s_{i}}^{\delta} \nabla_{s_{i}}^{\delta} I(x, y)=\left(-\frac{n-1}{16} r^{2}+o\left(r^{4}\right)\right) I(x, y)$,

2. $\Sigma_{i} \operatorname{div}\left(s_{i}\right) \nabla_{s_{i}}^{\delta} I(x, y)=0$.

Beweis. Den rein rechnerischen Beweis führen wir in mehreren Schritten.

1. Schritt. Die kovariante Ableitung $\nabla^{\mathscr{\delta}}$ im Spinorbündel wird durch die Formen $w_{i j}\left(s_{k}\right)=\left(\nabla_{s_{k}} s_{i}, s_{j}\right)$ des Levi-Civita-Zusammenhanges beschrieben. Daher berechnen wir zuerst für die Sphäre $S^{n}$ diese Zusammenhangsformen, wobei das Problem mit Hinblick auf den zu beweisenden Satz darin besteht, für sie Ausdrücke in Abhängigkeit vom geodätischen Abstand $r$ zu erhalten. Sei $S^{n}$ als Einheitssphäre in den euklidischen Raum $R^{n+1}$ eingebettet und $e_{1}, \cdots, e_{n+1}$ die Standartbasis dieses. Aus Symmetriegründen können wir $y=(0, \cdots, 0,1)$ und $s_{k}(y)=\left(y, e_{k}\right), 1 \leqslant k \leqslant n$, annehmen. Wir müssen $\nabla_{s_{k}} s_{i}$ in einem Punkt $x_{0}$ berechnen, dessen Koordinaten wir aus den gleichen Gründen

$$
x_{0}=\left(\sin t_{0}, 0, \cdots, 0, \cos t_{0}\right)
$$

setzen können. Die kovariante Ableitung ist in unserem Fall durch

$$
\left(\nabla_{s_{k}} s_{i}\right)\left(x_{0}\right)=s_{k} \cdot s_{i}-\left(s_{k}, s_{i}\right) x_{0}
$$

gegeben, wobei $s_{k} \cdot s_{i}$ die Differentiation von $s_{i}$ nach $s_{k}$ ist. Daraus erhalten wir

$$
w_{i j}\left(s_{k}\right)\left(x_{0}\right)=\left(s_{k} \cdot s_{i}, s_{j}\right)\left(x_{0}\right)
$$


und somit genügt es, den Vektor $s_{k} \cdot s_{i}$ zu berechnen. Sei vorerst $k$ größer als 1. Dann gilt $s_{k}\left(x_{0}\right)=\left(x_{0}, e_{k}\right)$ und wir erhalten für die durch $\gamma_{k}(0)=x_{0}$, $\dot{\gamma}_{k}(0)=s_{k}\left(x_{0}\right)$ eindeutig bestimmte Geodätische $\gamma_{k}(t)$ in $S^{n}$ die Formel

$$
\gamma_{k}(t)=\cos t x_{0}+\sin t e_{k} .
$$

Wir betrachten jetzt die Geodätische $\delta_{k}^{t}$, welche $y$ mit $\gamma_{k}(t)$ verbindet und durch die Formel

$$
\delta_{k}^{t}(s)=\left(\frac{\cos t}{\sin \alpha_{t}} \sin t_{0} \sin s, 0, \cdots, 0, \frac{\sin t}{\sin \alpha_{t}} \sin s, 0, \cdots, 0, \cos (s)\right)
$$

mit $\sin \alpha_{t}=\sqrt{\cos ^{2} t \sin ^{2} t_{0}+\sin ^{2} t}$ gegeben ist. Dessen Tangentialvektor im Punkt $s=0$ ist

$$
\dot{\delta}_{k}^{t}(0)=\left(\frac{\cos t}{\sin \alpha_{t}} \sin t_{0}, 0, \cdots, 0, \frac{\sin t}{\sin \alpha_{t}}, 0, \cdots, 0\right),
$$

und somit gilt

$$
\left(s_{i}(y), \dot{\delta}_{k}^{t}(0)\right)=0 \text { für } i \neq 1, k,
$$

(b) $\quad s_{1}(y)=\frac{\cos t}{\sin \alpha_{t}} \sin t_{0} \dot{\delta}_{k}^{t}(0)$

$$
+\frac{\sin t}{\sin \alpha_{t}}\left(\frac{\sin t}{\sin \alpha_{t}}, 0, \cdots, 0-\frac{\cos t}{\sin \alpha_{t}} \sin t_{0}, 0, \cdots, 0\right)
$$

mit

$$
\left(\dot{\delta}_{k}^{t}(0),\left(\frac{\sin t}{\sin \alpha_{t}}, 0, \cdots, 0,-\frac{\cos t}{\sin \alpha_{t}} \sin t_{0}, 0, \cdots, 0\right)\right)=0 .
$$

Weil $s_{i}\left(\gamma_{k}(t)\right)$ aus $s_{i}(y)=\left(y, e_{i}\right)$ durch Parallelverschiebung entlang $\delta_{k}^{t}$ entsteht, erhalten wir folgende Ausdrücke für $s_{i}\left(\gamma_{k}(t)\right)$ :

$$
s_{i}\left(\gamma_{k}(t)\right)=\left(\gamma_{k}(t), e_{i}\right), i \neq 1, k \text {. }
$$

$\left(b^{\prime}\right)$

$$
\begin{aligned}
s_{1}\left(\gamma_{k}(t)\right)= & \frac{\cos t}{\sin \alpha_{t}} \sin t_{0} \dot{\delta}_{k}^{t}\left(\alpha_{t}\right) \\
& +\frac{\sin t}{\sin \alpha_{t}}\left(\frac{\sin t}{\sin \alpha_{t}}, 0, \cdots, 0-\frac{\cos t}{\sin \alpha_{t}} \sin t_{0}, 0, \cdots, 0\right) .
\end{aligned}
$$

Aus $\left(s_{k} \cdot s_{i}\right)\left(x_{0}\right)=(d / d t) s_{i}\left(\gamma_{k}(t)\right)_{t=0}$ folgt nun

$$
\begin{gathered}
\left(s_{k} \cdot s_{i}\right)\left(x_{0}\right)=0 \text { für } i \neq 1, k, \\
\left(s_{k} \cdot s_{1}\right)\left(x_{0}\right)=\left(0, \cdots, 0,-\tan \left(t_{0} / 2\right), 0, \cdots, 0\right) .
\end{gathered}
$$


Dann ergibt sich unter Berücksichtigung von $t_{0}=r\left(x_{0}, y\right)=r_{0}$ :

$$
\begin{aligned}
& w_{i j}\left(s_{k}\right)=0, i \neq 1, k, \\
& w_{1 j}\left(s_{k}\right)=0, j \neq k, w_{1 k}\left(s_{k}\right)=-\tan \left(r_{0} / 2\right), \\
& w_{k j}\left(s_{k}\right)=0, j \neq 1, w_{k 1}\left(s_{k}\right)=-w_{1 k}\left(s_{k}\right)=\tan \left(r_{0} / 2\right) .
\end{aligned}
$$

Behandeln wir den Fall $k=1$ analog, so erhalten wir insgesamt folgendes Ergebnis:

$$
w_{j l}\left(s_{i}\right)\left(x_{0}\right)= \begin{cases}-\tan \left(r_{0} / 2\right), & j=1, l=i>1, \\ \tan \left(r_{0} / 2\right), & l=1, j=i>1, \\ 0 & \text { sonst. }\end{cases}
$$

2. Schritt. Wir zeigen $s_{i}\left(w_{j l}\left(s_{i}\right)\right)=0$. Da nur $w_{1 i}\left(s_{i}\right)$ verschieden von Null sind, genügt es $s_{i}\left(w_{1 i}\left(s_{i}\right)\right)=0 \mathrm{zu}$ zeigen. Der Abstand von $\gamma_{i}(t)=(\cos t$. $\left.\sin t_{0}, \cdots, \cos t, \cdots, \cos t \cdot \cos t_{0}\right)$ zu $y=(0, \cdots, 0,1)$ in $S^{n}$ beträgt

$$
r\left(\gamma_{i}(t), y\right)=\cos ^{-1}\left(\cos t \cdot \cos t_{0}\right)
$$

und somit gilt

$$
w_{1 i}\left(s_{i}\right)\left(\gamma_{i}(t)\right)=-\tan \left(\frac{1}{2} \cos ^{-1}\left(\cos t \cdot \cos t_{0}\right)\right),
$$

woraus $s_{i}\left(w_{1 i}\left(s_{i}\right)\right)\left(x_{0}\right)=\left.(d / d t)\left(w_{1 i}\left(s_{i}\right)\left(\gamma_{i}(t)\right)\right)\right|_{t=0}=0$ folgt.

3. Schritt. Der Schnitt $I(x, y)$ wurde dadurch definiert, daß wir in $\delta_{y}$ eine orthonormierte Basis $e_{\varepsilon}$ wählten, diese parallel im Spinorbündel nach $x$ verschoben und $I(x, y)=\Sigma_{\varepsilon} e_{\varepsilon}(x) \otimes e_{\varepsilon}(y)$ setzten. Weil das Reper von Vektorfeldern $\left(s_{1}, \cdots, s_{n}\right)$ als durch Parallelverschiebung von $y$ hervorgehend vorausgesetzt ist, haben die in Abschnitt 3 definierten Schnitte $\eta_{e}(x)=$ $\left[\hat{s}(x), u_{e}\right]$ die gleiche Eigenschaft. Wir können demnach $e_{e}(x)=\eta_{e}(x)$ annehmen. Aus der Formel für die kovariante Ableitung $\nabla^{\delta}$ erhalten wir dann

$$
\begin{aligned}
\sum_{i} \nabla_{s_{i}}^{\delta} \nabla_{s_{i}}^{\delta} e_{\varepsilon}\left(x_{0}\right)= & \sum_{i} \sum_{l<m} \frac{1}{2} s_{i}\left(w_{l m}\left(s_{i}\right)\right)\left(s_{l} s_{m}\right) \cdot e_{\varepsilon}\left(x_{0}\right) \\
& +\sum_{i} \sum_{l<m} \sum_{p<q} \frac{1}{4} w_{l m}\left(s_{i}\right) w_{p q}\left(s_{i}\right)\left(s_{p} s_{q} s_{l} s_{m}\right) \cdot e_{\varepsilon}\left(x_{0}\right) .
\end{aligned}
$$

Aus den Formeln für $w_{i j}\left(s_{k}\right)$ folgt dann unmittelbar

$$
\sum_{i} \nabla_{s_{i}}^{\delta} \nabla_{s_{i}}^{\delta} e_{\varepsilon}\left(x_{0}\right)=-\frac{n-1}{4} \tan ^{2} \frac{r_{0}}{2} e_{\varepsilon}\left(x_{0}\right),
$$

und somit

$$
\sum_{i} \nabla_{s_{i}}^{\delta} \nabla_{s_{i}}^{\delta} I(x, y)=\left(-\frac{n-1}{16} r^{2}+o\left(r^{4}\right)\right) I(x, y) .
$$


4. Schritt. Aus $\operatorname{div}\left(s_{i}\right)=\sum_{j=1}^{n} w_{i j}\left(s_{j}\right)$ ergibt sich nun

$$
\operatorname{div}\left(s_{i}\right)= \begin{cases}0, & i \neq 1, \\ -(n-1) \tan \left(r_{0} / 2\right), & i=1,\end{cases}
$$

und somit

$$
\begin{aligned}
\sum_{i} \operatorname{div}\left(s_{i}\right) \nabla_{s_{i}}^{\delta} e_{\varepsilon} & =-(n-1) \tan \left(r_{0} / 2\right) \nabla_{s_{i}}^{\delta} e_{\varepsilon} \\
& =-(n-1) \tan \left(r_{0} / 2\right) \sum_{l<m} \frac{1}{2} w_{l m}\left(s_{1}\right)\left(s_{l} s_{m}\right) \cdot e_{\varepsilon}=0 .
\end{aligned}
$$

Wir erhalten daher die zweite Behauptung

$$
\sum_{i} \operatorname{div}\left(s_{i}\right) \nabla_{s_{i}}^{\delta} I(x, y)=0 .
$$

Bemerkung. Ist $\Psi=g I$ ein Schnitt über $U_{\varepsilon}$, wobei $g$ nur vom Abstand $r=r(x, y)$ abhängt, so gilt $\Sigma_{i} \nabla_{s_{i}}^{\delta} I \circ s_{i}(\Psi)=0$. Der Beweis ergibt sich aus folgender einfacher Rechnung

$$
\sum_{i} \nabla_{s_{i}}^{\delta} I \circ s_{i}(\Psi)=\sum_{i} s_{i}(g) \nabla_{s_{i}}^{\delta} I \circ I=\nabla_{\mathrm{grad} g}^{\delta} I .
$$

Der Gradient von $g$ grad $g=g^{\prime} \cdot \dot{\gamma}(r)$ ist parallel zum Radialfeld und I wurde als parallel verschoben in dessen Richtung definiert. Daher gilt $\nabla_{\text {grad } g}^{\delta} I=0$.

Satz 4. Im Fall der Sphäre $S^{n}$ gilt für eine nur vom Abstand $r$ abhängende Funktion $g=g(r)$ die Formel

$$
D^{2}(g I)=\left\{\left(\frac{1}{4} n(n-1)+\frac{n-1}{16} r^{2}+o\left(r^{4}\right)\right) g+\Delta(g)\right\} I .
$$

Insbesondere haben die zur Bestimmung der Parametrix $W$ eingeführten Schnitte $U_{j}$ die "Diagonalform" $U_{j}=g_{j} I$, in welcher $g_{j}=g_{j}(r)$ nur vom Abstand $r$ abhängige Funktionen sind und welche induktiv gemäß

$$
\begin{aligned}
& g_{0}(r)=\theta^{-1 / 2}(r) \\
& g_{j}(r)=-\theta^{-1 / 2}(r) \int_{0}^{1} \theta^{1 / 2}(r \xi) \xi^{j-1}\left\{\left(\frac{1}{4} n(n-1)+\frac{n-1}{16} r^{2} \xi^{2}+o\left(r^{4}\right)\right)\right. \\
& \left.\left.\cdot g_{j-1}(r \xi)+\Delta\left(g_{j-1}\right)(r \xi)\right\} d \xi\right\}
\end{aligned}
$$

gegeben sind.

Beweis. Die erste Behauptung folgt mit Satz 3 und der obigen Bemerkung aus

$$
D^{2}(\Psi)=\frac{1}{4} R \Psi+\Delta(\Psi)-\left(\sum_{i} \nabla_{s_{i}}^{\delta} \nabla_{s_{i}}^{\delta} I+\operatorname{div}\left(s_{i}\right) \nabla_{s_{i}}^{\delta} I\right) \circ \Psi-2 \sum_{i} \nabla_{s_{i}}^{\delta} I \circ s_{i}(\Psi)
$$

während sich die Rekursionsformel für $g_{j}$ aus derjenigen für $U_{j}$ und dem 
angegebenen Ausdruck

$$
D^{2}(g I)=\left\{\left(\frac{1}{4} n(n-1)+\frac{n-1}{16} r^{2}+o\left(r^{4}\right)\right) g+\Delta(g)\right\} I
$$

ergibt. q.e.d.

Wir wenden uns nun der Berechnung der Koeffizienten $d_{1}$ und $d_{2} \mathrm{zu}$. Dazu verwenden wir folgende auf der Sphäre $S^{n}$ geltende Formeln (vgl. [2])

$$
\begin{aligned}
\theta(r) & =\left(\frac{\sin r}{r}\right)^{n-1}, \\
g_{0}(r) & =\left(\frac{\sin r}{r}\right)^{-(n-1) / 2}=1+\frac{n-1}{12} r^{2}+\frac{(n-1)(5 n-1)}{1440} r^{4}+o\left(r^{6}\right), \\
\frac{\theta^{\prime}}{\theta}(r) & =-\frac{n-1}{3} r+o\left(r^{3}\right),
\end{aligned}
$$

sowie folgende Formel für den Laplace-Operator einer nur vom Abstand abhängenden Funktion $g$

$$
\Delta(g)=-g^{\prime \prime}-g^{\prime}\left(\frac{\theta^{\prime}}{\theta}+\frac{n-1}{r}\right) .
$$

Setzen wir dies in die Rekursionsformel für $g_{1}$ ein, so erhalten wir

$$
g_{1}(r)=-\frac{1}{12} R+\frac{r^{2}}{2160}\left(-15 n^{3}+18 n^{2}-12 n+9\right)+o\left(r^{3}\right)
$$

und daher gilt

$$
U_{1}(y, y)=g_{1}(o)=-\frac{1}{12} R \cdot I(y, y)
$$

Wir hatten $d_{1}$ bereits in der Form $d_{1}=k(n) \cdot \int_{X^{n}} R(x) d x$ dargestellt. Setzen wir nun $X^{n}=S^{n}$, so erhalten wir $k(n)=-\operatorname{dim} \mathcal{S} / 12$, also

$$
d_{1}=-\frac{\operatorname{dim} \mathcal{S}}{12} \int_{X^{n}} R(x) d x .
$$

Weiterhin ergibt sich

$$
\begin{aligned}
g_{2}(o) & =\frac{1}{4320}\left(15 n^{4}-54 n^{3}+21 n^{2}+18 n\right) \\
& =\frac{1}{4320}\left(15 \cdot n^{2}(n-1)^{2}-21 \cdot 2 n(n-1)-24 \cdot n(n-1)^{2}\right),
\end{aligned}
$$

und somit

$$
\begin{aligned}
\operatorname{Tr} U_{2} & =\operatorname{dim} \delta \cdot g_{2}(o) \\
& =\frac{\operatorname{dim} \delta}{4320}\left(15 n^{2}(n-1)^{2}-21 \cdot 2 n(n-1)-24 n(n-1)^{2}\right)
\end{aligned}
$$


Andererseits hatten wir $\operatorname{Tr} U_{2}$ in der Form

$$
\operatorname{Tr} U_{2}=\alpha(n) R^{2}+\beta(n)\|\Re\|^{2}+\gamma(n) \rho^{2}+\delta(n) \Delta R
$$

dargestellt, woraus im Fall $X=S^{n}$

$$
a(n) n^{2}(n-1)^{2}+b(n) 2 n(n-1)+c(n) n(n-1)^{2}=0
$$

mit

$$
\begin{aligned}
& a(n)=\frac{\alpha(n)}{\operatorname{dim} \delta}-\frac{15}{4320}, \\
& b(n)=\frac{\beta(n)}{\operatorname{dim} \delta}+\frac{21}{4320}, \\
& c(n)=\frac{\gamma(n)}{\operatorname{dim} \delta}+\frac{24}{4320}
\end{aligned}
$$

folgt. Wir wollen nun $a(n) \equiv 0, b(n) \equiv 0, c(n) \equiv 0$ für $n \geqslant 3$ schließen. Zu diesem Ziel benötigen wir eine weitere Serie von Test-Mannigfaltigkeiten, welche wir in der Form $X^{k}=S^{n} \times S^{m}(n+m=k)$ wählen. Weil die Metrik von $S^{n} \times S^{m}$ das Produkt der Metriken von $S^{n}$ und $S^{m}$ ist, erhält man für diesen Raum unter Verwendung der auf $S^{n}$ und $S^{m}$ bereits berechneten Größen die Zusammenhangskoeffizienten $w_{i j}\left(s_{i}\right)$ und den Schnitt $\Sigma_{i} \nabla_{s_{i}}^{\delta} \nabla_{s_{i}}^{\delta} I+\operatorname{div}\left(s_{i}\right) \nabla_{s_{i}}^{\delta} I$, welcher wiederum ein Vielfaches von $I$ ist. Daraus bekommt man in Analogie zu der für $X^{n}=S^{n}$ durchgeführten Rechnungden Wert

$$
\begin{aligned}
g_{2}^{S^{n} \times S^{m}}(o): & \\
g_{2}^{S^{n} \times S^{m}}(o)= & \frac{1}{4320}\left[15(n(n-1)+m(m-1))^{2}-21(2 n(n-1)\right. \\
& \left.+2 m(m-1))-24\left(n(n-1)^{2}+m(m-1)^{2}\right)\right] .
\end{aligned}
$$

Mit den oben eingeführten Bezeichnungen erhalten wir

$$
\begin{aligned}
0= & a(n+m)(n(n-1)+m(m-1))^{2}+2 b(n+m)(n(n-1)+m(m-1)) \\
& +c(n+m)\left(n(n-1)^{2}+m(m-1)^{2}\right) .
\end{aligned}
$$

Setzen wir jetzt $n=\lambda$ und $m=k-\lambda$, so entsteht ein Polynom 4. Grades in $\lambda$ mit den Nullstellen $\lambda=0,1, \cdots, k$. Daraus schließt man für $k \geqslant 4$ leicht $a(k)=b(k)=c(k)=0$. Der Fall dritter Dimension muß etwas anders behandelt werden, weil wir drei Unbekannte $a(3)=a, b(3)=b, c(3)=c$, aber nur zwei Test-Mannigfaltigkeit $S^{3}, S^{2} \times S^{1}$ und somit nur zwei Beziehungen zwischen ihnen haben

$$
3 a+b+c=0,2 a+2 b+c=0 .
$$

Dafür besteht aber in einem 3-dimensionalen Riemannschen Raum die Beziehung $R^{2}-4 \rho^{2}+\|\Re\|^{2}=0$ ! 
Aus ihr erhalten wir

$$
d_{2}=\int_{X^{3}} \alpha R^{2}+\beta\|\Re\|^{2}+\gamma \rho^{2}=\int_{X^{3}}(\beta-\alpha)\|\Re\|^{2}+(\gamma+4 \alpha) \rho^{2} .
$$

Aus der Definition der Größen $a, b$ und $c$ folgt unmittelbar

$$
\beta-\alpha=2 b-2 a-\frac{72}{4320}, \gamma+4 \alpha=2 c+8 a+\frac{72}{4320},
$$

woraus man unter Verwendung von (2) $\beta-\alpha=-72 / 4320$ und $\gamma+4 \alpha=$ $72 / 4320$ schließt. Dann aber gilt

$$
d_{2}=\int_{X^{3}}(\beta-\alpha)\|\Re\|^{2}+(\gamma+4 \alpha) \rho^{2}=\frac{2}{4320} \int_{X^{3}}-36\|\Re\|^{2}+36 \rho^{2}
$$

und eine nochmalige Anwendung von $R^{2}-4 \rho^{2}+\|\Re\|^{2}=0$ ergibt die Formel

$$
d_{2}=\frac{\operatorname{dim} \delta}{4320} \int_{X^{3}} 15 R^{2}-21\|\Re\|^{2}-24 \rho^{2} .
$$

Das Ergebnis der bisherigen Überlegungen zusammenfassend formulieren wir

Theorem. Sei $X^{n}(n \geqslant 3)$ eine geschlossene Spin-Mannigfaltigkeit und $D$ der im Spinorbündel wirkende Dirac-Operator. Die Zeta-Funktion $\zeta(t)$ des Operators $D^{2}$ besitzt an der Stelle $t=0$ die asymptotische Entwicklung

mit

$$
\zeta(t) \sim(4 \pi t)^{-n / 2} \sum_{j=0}^{\infty} d_{j} t^{j}
$$

$$
\begin{aligned}
& d_{0}=\operatorname{dim} \delta \cdot \operatorname{Vol}(X), \\
& d_{1}=-\frac{\operatorname{dim} \delta}{12} \int_{X^{n}} R(x) d x, \\
& d_{2}=\frac{\operatorname{dim} \delta}{4320} \int_{X^{n}}\left(15 R(x)^{2}-21\|\Re(x)\|^{2}-24 \rho(x)^{2}\right) d x .
\end{aligned}
$$

\section{Einige Anwendungen der Asymptotenentwicklung}

\section{der Zeta-Funktion von $D^{2}$}

In diesem Abschnitt der Arbeit wenden wir uns der Frage zu, welche Größen der Riemannschen Mannigfaltigkeit das Spektrum $\operatorname{Spec}^{\Delta}$ des Laplace-Operators und das Spektrum $\operatorname{Spec}^{D^{2}}$ des Operators $D^{2}$ gemeinsam bestimmen. Aus der Asymptotenentwicklung der Zeta-Funktion $\zeta^{D^{2}}(t)$ des Operators $D^{2}$ folgt mit dem oben bewiesenen Theorem, daß die Integrale

$$
\int_{X^{n}} R(x) d x, \quad \int_{X^{n}}\left(15 R(x)^{2}-21\|\Re(x)\|^{2}-24 \rho(x)^{2}\right) d x,
$$


sowie das Volumen und die Dimension der Mannigfaltigkeit $X^{n}$ durch Spec $^{D^{2}}$ determiniert werden. Andererseits bestimmt das Spektrum $\operatorname{Spec}^{\Delta}$ des Laplace-Operators außer dem Volumen und der Dimension der Mannigfaltigkeit die Integrale

$$
\int_{X^{n}} R(x) d x, \quad \int_{X^{n}}\left(5 R(x)^{2}+2\|\Re(x)\|^{2}-2 \rho(x)^{2}\right) d x,
$$

(siehe [2]). Durch eine Kombination dieser Ausdrücke erhalten wir, daß für eine geschlossene Spin-Mannigfaltigkeit die Größen

$$
\int_{X^{n}} 3 R^{2}-2 \rho^{2}, \int_{X^{n}} 3\|\Re\|^{2}+2 \rho^{2}, \int_{X^{n}}\|\Re\|^{2}+R^{2}
$$

durch die beiden Spektren $\operatorname{Spec}^{\Delta}$ und $\operatorname{Spec}^{D^{2}}$ zusammen determiniert werden.

Satz 5. Seien $X^{4}$ eine 4-dimensionale, geschlossene Spin-Mannigfaltigkeit konstanter Skalarkrümmung $R$, und $\bar{X}$ eine geschlossene Spin-Mannigfaltigkeit konstanter Skalarkrümmung $\bar{R}$ mit $\operatorname{Spec}^{D^{2}}\left(X^{4}\right)=\operatorname{Spec}^{D^{2}}(\bar{X})$ und $\operatorname{Spec}^{\Delta}\left(X^{4}\right)=$ $\operatorname{Spec}^{\Delta}(\bar{X})$. Dann sind die Eulerschen Charakteristiken $\chi\left(X^{4}\right)$ und $\chi(\bar{X})$ der Mannigfaltigkeit $X^{4}$ und $\bar{X}$ gleich.

Bemerkung. Bereits aus $\operatorname{Spec}^{\Delta}\left(X^{4}\right)=\operatorname{Spec}^{\Delta}(\bar{X})$ oder $\operatorname{Spec}^{D^{2}}\left(X^{4}\right)=$ $\operatorname{Spec}^{D^{2}}(\bar{X})$ folgt $R=\bar{R}, \operatorname{dim} \bar{X}=\operatorname{dim} X^{4}=4$ und $\operatorname{Vol}(X)=\operatorname{Vol}(\bar{X})$.

Beweis. Aus dem Satz von Gauß-Bonet für 4-dimensionale Mannigfaltigkeit (vgl. [2]) erhalten wir

$$
\begin{aligned}
\chi\left(X^{4}\right) & =\frac{1}{32 \pi^{2}} \int_{X^{4}}\left(R^{2}+\|\Re\|^{2}-4 \rho^{2}\right) \\
& =\frac{1}{32 \pi^{2}}\left\{\int_{X^{4}}\left(R^{2}+\|\Re\|^{2}\right)+2 \int_{X^{4}}\left(3 R^{2}-2 \rho^{2}\right)-6 \int_{X^{4}} R^{2}\right\} .
\end{aligned}
$$

Die beiden ersten Integrale sind nach (3) durch die beiden Spektren determiniert, während $\int_{X} R^{2}=\int_{\bar{X}} \bar{R}^{2}$ aus $R=\bar{R}$ const und $\operatorname{Vol}(X)=\operatorname{Vol}(\bar{X})$ folgt. q.e.d.

Ist $X^{4}$ eine reell-4-dimensionale komplexe Kähler-Mannigfaltigkeit, so gilt für deren Signatur sign $\left(X^{4}\right)$ und deren arithmetisches Geschlecht a $\left(X^{4}\right)$ (vgl. [3])

$$
\begin{aligned}
\operatorname{sign}\left(X^{4}\right) & =-\frac{1}{48 \pi^{2}} \int\|\Re\|^{2}-2 \rho^{2}, \\
a\left(X^{4}\right) & :=\frac{1}{12}\left(c_{1}^{2}+c_{2}\right)\left[X^{4}\right]=\frac{1}{384 \cdot \pi^{2}} \int\|\Re\|^{2}-8 \rho^{2}+3 R^{2} .
\end{aligned}
$$

Satz 6. Seien $X^{4}, \bar{X}^{4}$ reell-4-dimensionale, geschlossene komplexe KählerMannigfaltigkeit mit Spin-Struktur und konstanter Skalarkrümmung. Aus $\operatorname{Spec}^{\Delta}(x)=\operatorname{Spec}^{\Delta}(\bar{X})$ und $\operatorname{Spec}^{D^{2}}(X)=\operatorname{Spec}^{D^{2}}(\bar{X})$ folgt die Gleichheit der 
Signaturen $\operatorname{sign}(X)=\operatorname{sign}(\bar{X})$ und der arithmetischen Geschlechte $a(X)=$ $a(\bar{X})$.

Beweis. Aus dem oben angegebenen Ausdruck für $a(X)$ erhalten wir

$$
\begin{aligned}
a(X) & =\frac{1}{384 \pi^{2}} \int_{X^{4}}\|\Re\|^{2}-8 \rho^{2}+3 R^{2} \\
& =\frac{1}{384 \pi^{2}}\left\{\int_{X^{4}}\left(\|\Re\|^{2}+R^{2}\right)+4 \int_{X^{4}}\left(3 R^{2}-2 \rho^{2}\right)-10 \int_{X^{4}} R^{2}\right\}
\end{aligned}
$$

und schließen wie im Beweis von Satz 5. Der Beweis für die Signatur verläuft analog. q.e.d.

Wir wenden uns nun Mannigfaltigkeit mit konstanter Schnittkrümmung zu und beweisen

Satz 7. Es seien $X^{n}$ und $\bar{X}^{n}$ geschlossene Spin-Mannigfaltigkeit, für welche die Spektren der Laplace-und Dirac-Operatoren $\operatorname{Spec}^{\Delta}$ und $\operatorname{Spec}^{D^{2}}$ gleich sind. Hat $X^{n}$ eine konstante Schnittkrümmung $\sigma$, so istdie Schnittkrümmung $\bar{\sigma}$ von $\bar{X}^{n}$ ebenfalls konstant und gleich $\sigma$.

Beweis. Da $X^{n}$ ein Riemannscher Raum mit konstanter Schnittkrümmung ist, gilt $\|\Re\|^{2}=2 /(n-1) \rho^{2}$ und $\rho^{2}=R^{2} / n$, während wir für $\bar{X}^{n}$ die Ungleichungen $\|\bar{\Re}\|^{2} \geqslant 2 /(n-1) \bar{\rho}^{2}$ und $\bar{\rho}^{2} \geqslant \bar{R}^{2} / n$ haben. Wegen $R=n(n-$ 1)o folgt, daß $R$ konstant ist. Aus der Gleichheit der Spektren Spec ${ }^{\Delta}$ und $\operatorname{Spec}^{D^{2}}$ erhalten wir

$$
\int_{\bar{X}}\|\bar{\Re}\|^{2}+\bar{R}^{2}=\int_{X}\|\Re\|^{2}+R^{2}, \quad \int_{\bar{X}} \bar{R}=\int_{X} R,
$$

woraus wir mittels

$$
\begin{aligned}
\left(\frac{2}{n(n-1)}+1\right) \int_{\bar{X}} \bar{R}^{2} & \leqslant \int_{\bar{X}}\|\bar{\Re}\|^{2}+\bar{R}^{2}=\int_{X}\|\Re\|^{2}+R^{2} \\
& =\left(\frac{2}{n(n-1)}+1\right) \int_{X} R^{2}
\end{aligned}
$$

die Ungleichung $\int_{\bar{X}} \bar{R}^{2} \leqslant \int_{X} R^{2}$ und somit

$$
\operatorname{Vol}(\bar{X}) \int_{\bar{X}} \bar{R}^{2} \leqslant \operatorname{Vol}(X) \int_{X} R^{2}=\left(\int_{X} R\right)^{2}=\left(\int_{\bar{X}} \bar{R}\right)^{2}
$$

bekommen. Eine Anwendung der Cauchy-Schwarzschen Ungleichung ergibt dann

$$
\left(\int_{\bar{X}} \bar{R}\right)^{2} \leqslant \int_{\bar{X}} \bar{R}^{2} \cdot \int_{\bar{X}} 1^{2}=\int_{\bar{X}} \bar{R}^{2} \cdot \operatorname{Vol}(\bar{X})<\left(\int_{\bar{X}} \bar{R}\right)^{2}
$$

Daher tritt in der Cauchy-Schwarzschen Ungleichung Gleichheit ein, also ist $\bar{R}$ konstant und wegen $\int_{\bar{X}} \bar{R}=\int_{X} R$ gleich $R$. Da die in (1) angeführten 
Integrale durch die Spektren $\operatorname{Spec}^{\Delta}$ und $\operatorname{Spec}^{D^{2}}$ determinert werden, erhalten wir aus $R=\bar{R}$

$$
\int_{X} \rho^{2}=\int_{\bar{X}} \bar{\rho}^{2}, \quad \int_{X}\|\Re\|^{2}=\int_{\bar{X}}\|\bar{\Re}\|^{2}
$$

Dann aber gilt

$$
\frac{2}{n-1} \int_{X} \rho^{2}=\frac{2}{n-1} \int_{\bar{X}} \bar{\rho}^{2} \leqslant \int_{\bar{X}}\|\bar{\Re}\|^{2}=\int_{X}\|\Re\|^{2}=\frac{2}{n-1} \int_{X} \rho^{2},
$$

und somit $2 /(n-1) \bar{\rho}^{2}=\|\bar{\Re}\|^{2}$. Diese Bedingung ist jedoch notwendig und hinreichend dafür, daß $\bar{X}$ eine konstante Schnittkrümmung $\bar{\sigma}$ hat. Die Gleichheit $\bar{\sigma}=\sigma$ folgt aus $\bar{R}=R$ unmittelbar.

Folgerung 4. Seien $X^{n}$ eine geschlossene Spin-Mannigfaltigkeit mit konstanter Schnittkrümmung, und $\bar{X}^{n}$ eine geschlossene Spin-Mannigfaltigkeit. Sind die Spektren der Operatoren $\Delta$ und $D^{2}$ auf $X^{n}$ und $\bar{X}^{n}$ gleich, so sind $X^{n}$ und $\bar{X}^{n}$ lokal isometrisch.

Bekanntlicherweise sind die Sphären $S^{2}, S^{3}, S^{4}$ und die reell-projektiven Räume $\mathbf{P}^{2}(\mathbf{R}), \mathbf{P}^{3}(\mathbf{R}), \mathbf{P}^{4}(\mathbf{R})$ durch das Spektrum des Laplace-Operators als Riemannsche Mannigfaltigkeit bestimmt (vgl. [2]). Wir zeigen nun

Folgerung 5. Ist $X^{n}$ eine geschlossene zusammenhängende Spin-Mannigfaltigkeit mit $\operatorname{Spec}^{\Delta}\left(X^{n}\right)=\operatorname{Spec}^{\Delta}\left(S^{n}\right)$ und $\operatorname{Spec}^{D^{2}}\left(X^{n}\right)=\operatorname{Spec}^{D^{2}}\left(S^{n}\right)$, so ist $X^{n}$ isometrisch $z u S^{n}$.

Beweis. Aus den Voraussetzungen ergibt sich, daß $X^{n}$ konstante positive Schnittkrümmung $\sigma>o$ hat. Ein solcher Raum wird dann von der Sphäre $S^{n}$ überlagert: $X^{n}=S^{n} / \Gamma\left(\operatorname{vgl}\right.$. [15]). Aus $\operatorname{Vol}\left(X^{n}\right)=\operatorname{Vol}\left(S^{n}\right)$ und $\operatorname{Vol}\left(X^{n}\right)=$ $1 / \operatorname{card}(\Gamma)$. $\operatorname{Vol}\left(S^{n}\right)$ erhalten wir dann, daß $\Gamma$ nur aus der Identität besteht und somit $S^{n}=X^{n}$ ist. q.e.d.

Mit dem gleichen Argument erhält man

Folgerung 6. Ist $X^{4(k+3)}$ eine geschlossene, zusammenhängende Spin-Mannigfaltigkeit und $\operatorname{Spec}^{\Delta}\left(X^{4(k+3)}\right)=\operatorname{Spec}^{\Delta}\left(\mathbf{P}^{4(k+3)}(\mathbf{R})\right), \quad \operatorname{Spec}^{D^{2}}\left(X^{4(k+3)}\right)=$ $\operatorname{Spec}^{D^{2}}\left(\mathbf{P}^{4(k+3)}(\mathbf{R})\right)$, so ist $X^{4(k+3)}$ isometrisch zum reell-projektiven Raum $\mathbf{P}^{4(k+3)}(\mathbf{R})$.

\section{Berechnung der Koeffizienten $d_{0}, d_{1}, d_{2}$ nach einer Methode von P. B. Gilkey}

P. B. Gilkey (vgl. [6]) hat in Auswertung der Seeley-Formeln die Koeffizienten $a_{k}\left(P P^{*}\right)=\int_{X} \mu_{k}$ in der Asymptotenentwicklung der Zeta-Funktion des Operators $P P^{*}$ für den Fall angegeben, daß $P P^{*}$ ein Operator 2. Ordnung mit dem Symbol $\sigma(x, v)=-\|v\|$ ist. Dann nämlich können die $\mu_{k}$ in der Form

$$
\mu_{k}(x)=\operatorname{trace} E_{k}(x)
$$


dargestellt werden, wobei $E_{k}$ ein $\operatorname{Schnitt~im~} \operatorname{Bündel~} \operatorname{Hom}(E, E)$ ist. Die ersten Homomorphismen $E_{0}, E_{1}, E_{2}$ sind durch die Formeln

$$
\begin{aligned}
E_{0}=(4 \pi)^{-n / 2} \operatorname{Id}_{E}, \\
E_{1}=(4 \pi)^{-n / 2}\left(H+\frac{1}{6} R \cdot \operatorname{Id}_{E}\right), \\
E_{2}=(4 \pi)^{-n / 2}\left\{\left(\frac{1}{30} \Delta R+\frac{1}{72} R^{2}-\frac{1}{180} \rho^{2}+\frac{1}{180}\|\Re\|^{2}\right) \cdot \operatorname{Id}_{E}\right. \\
\left.\quad+\frac{1}{6} R H+\frac{1}{2} H^{2}+\frac{1}{12} W_{i j} W_{i j}+\frac{1}{6} \Delta H\right\}
\end{aligned}
$$

gegeben. Die Homomorphismen $H, W_{i j}$ aus $\operatorname{Hom}(E, E)$ sind dabei folgendermaßen zu bestimmen; Dem Operator $P P^{*}$ wird eine kovariante Ableitung $\nabla^{E}$ im Vektorbündel $E$ so zugeordnet, daß

$$
P P^{*}=-H-\sum_{i} \nabla_{s_{i}}^{E} \nabla_{s_{i}}^{E}-\sum_{i} \operatorname{div}\left(s_{i}\right) \nabla_{s_{i}}^{E}
$$

ist. $W_{i j}$ ergibt sich dann aus

$$
W_{i j}=\nabla_{s_{i}}^{E} \nabla_{s_{j}}^{E}-\nabla_{s_{j}}^{E} \nabla_{s_{i}}^{E}-\nabla_{\left[s_{i}, s_{j}\right]}^{E}
$$

Sei nun $E=\delta$ das Spinorbündel und $\nabla^{\complement}$ die kovariante Ableitung in diesem. Aus

$$
D^{2}=\frac{1}{4} R-\sum_{i} \nabla_{s_{i}}^{\delta} \nabla_{s_{i}}^{\delta}-\sum_{i} \operatorname{div}\left(s_{i}\right) \nabla_{s_{i}}^{\delta}
$$

ergibt sich dann, daß die kovariante Ableitung $\nabla^{\mathcal{\delta}}$ zur Auswertung dieser Gilkey-Formeln benutzt werden kann und $H=-\frac{1}{4} R$ gilt. Die Koeffizienten $d_{0}$ und $d_{1}$ in der Asymptotenentwicklung der Zeta-Funktion des Operators $D^{2}$ erhalten wir dann aus

$$
\begin{aligned}
& d_{0}=(4 \pi)^{n / 2} \int_{X} \mu_{0}=\int_{X} \operatorname{Tr} I d y=\operatorname{dim} \delta \cdot \operatorname{Vol}(X), \\
& d_{1}=(4 \pi)^{n / 2} \int_{X} \mu_{1}=\int_{X}\left(H+\frac{1}{6} R\right) \cdot \operatorname{Tr} I d y=-\frac{\operatorname{dim} \delta}{12} \int_{X} R(x) d x .
\end{aligned}
$$

Für die Berechnung des Koeffizienten $d_{2}$ benötigen wir die Spuren der Homomorphismen $W_{i j} \cdot W_{i j}$. Nun gilt

$$
W_{i j}=\nabla_{s_{i}}^{\delta} \nabla_{s_{i}}^{\delta}-\nabla_{s_{j}}^{\delta} \nabla_{s_{i}}^{\delta}-\nabla_{\left[s_{i}, s_{j}\right]}^{\delta}=\frac{1}{2} \sum_{l<m} R_{i j l m} s_{l} s_{m},
$$

wobei wir $s_{1}, s_{m}$ mittels der Clifford-Multiplikation als einen Homomorphismus im Spinorbündel auffassen, woraus unter Verwendung einer orthonormierten Basis $e_{i} \in \mathbf{R}^{n}$ mit $s_{i}=\left[s_{i}, e_{i}\right]$

$$
\operatorname{Trace}\left(W_{i j} W_{i j}\right)=\frac{1}{4} \sum_{l<m} \sum_{p<q} R_{i j l m} R_{i j p q} \operatorname{Trace}\left(e_{1} e_{m} e_{p} e_{q}\right)
$$


folgt. Die Wirkung der Produkte $e_{1} e_{m}$ auf dem Spin-Modul $\Delta_{n}$ wurde in [4] beschrieben. In $\Delta_{n}$ führt man eine Basis $u\left(\varepsilon_{1}, \cdots, \varepsilon_{k}\right)$ mit $n=2 k, 2 k+1$ und $\varepsilon_{i}= \pm 1$ ein, für welche mit

$$
\alpha(j)= \begin{cases}1 & \text { falls } j \text { ungerade } \\ 2 & \text { falls } j \text { gerade }\end{cases}
$$

die nachstehenden Formeln abgeleitet wurden:

1. Ist $[l-1 / 2]<[m-1 / 2]$ und $m<2 k+1$ :

$$
\begin{aligned}
\left(e_{l} e_{m}\right) & \cdot u\left(\varepsilon_{1}, \cdots, \varepsilon_{k}\right)=C_{l m} \varepsilon_{k-[m-1 / 2]}^{\alpha(m+1)} \cdot \varepsilon_{k-[l-1 / 2]}^{\alpha(1)} \cdot \varepsilon_{k-[m-1 / 2]+1} \\
& \cdots \varepsilon_{k-[l-1 / 2]-1} \cdot u\left(\varepsilon_{1}, \cdots,-\varepsilon_{k-[m-1 / 2]}, \cdots,-\varepsilon_{k-[l-1 / 2]}, \cdots, \varepsilon_{k}\right) .
\end{aligned}
$$

2. Ist $l<m<2 k+1$ und $[l-1 / 2]=[m-1 / 2]$ :

$$
\left(e_{l} e_{m}\right) \cdot u\left(\varepsilon_{1}, \cdots, \varepsilon_{k}\right)=i \cdot \varepsilon_{k-[l-1 / 2]} \cdot u\left(\varepsilon_{1}, \cdots, \varepsilon_{k}\right) .
$$

3. Ist $l<2 k+1, m=2 k+1$ :

$$
\begin{aligned}
& \left(e_{l} e_{m}\right) \cdot u\left(\varepsilon_{1}, \cdots, \varepsilon_{k}\right)=(-1)^{n-[l+1 / 2]+l_{i} \alpha(l)+1} \cdot \varepsilon_{1} \cdots \varepsilon_{k-[l+1 / 2]} \\
& \cdot \varepsilon_{k-[l-1 / 2]}^{\alpha(l)} \cdot u\left(\varepsilon_{1}, \cdots,-\varepsilon_{k-[l-1 / 2]}, \cdots, \varepsilon_{k}\right) .
\end{aligned}
$$

Aus diesen Formeln ergibt sich:

$$
\begin{aligned}
\operatorname{Tr}\left(e_{i} e_{j}\right) & =0, \quad i<j, \\
\operatorname{Tr}\left(e_{i} e_{j} e_{l} e_{m}\right) & =0, \quad i<j<l<m,
\end{aligned}
$$

und wir erhalten

$$
\begin{aligned}
\operatorname{Trace}\left(W_{i j} W_{i j}\right) & =\frac{1}{4} \sum_{l<m} R_{i j l m} R_{i j l m} \operatorname{Trace}\left(e_{l} e_{m} e_{l} e_{m}\right) \\
& =-\frac{\operatorname{dim} \delta}{4} \sum_{l<m} R_{i j l m}^{2}=-\frac{\operatorname{dim} \delta}{8} \sum_{l, m} R_{i j l m}^{2}, \\
\operatorname{Trace}\left(W_{i j} W_{i j}\right) & =-\frac{\operatorname{dim} \delta}{8}\|\Re\|^{2} .
\end{aligned}
$$

Setzen wir diesen Wert in die Formel für $E_{2}$ ein, so ergibt sich

$$
\begin{aligned}
d_{2} & =(4 \pi)^{n / 2} \int_{X} \mu_{2}=\int_{X} \operatorname{Tr} E_{2} \\
& =\frac{\operatorname{dim} \delta}{4320} \int_{X}\left(15 R(x)^{2}-21\|\Re(x)\|^{2}-24 \rho(x)^{2}\right) d x .
\end{aligned}
$$

\section{Literatur}

[1] M. F. Atiyah, R. Bott \& V. K. Patodi, On the heat equation and the index theorem, Invent. Math. 19 (1973) 279-330.

[2] M. Berger, P. Gauduchon \& E. Mazet, Le spectre d'une varieté Riemannienne, Lecture Notes in Math. Vol. 194, Springer, Berlin, 1971. 
[3] H. Donnelly, Topology and Einstein Kaehler metrics, J. Differential Geometry 11 (1976) 259-264.

[4] Th. Friedrich \& S. Sulanke, Ein Kriterium für die formale Selbstadjungiertheit des Dirac-Operators, Colloq. Math. 40 (1979) 239-247.

[5] M. P. Gaffney, Asymptotic distributions associated with the Laplacian for forms, Comm. Pure Appl. Math. 11 (1958) 535-545.

[6] B. P. Gilkey, The spectral geometry of real and complex manifolds, Proc. Symp. Pure Math. Vol. 27, Amer. Math. Soc. 1975, 265-280.

[7] N. Hitchin, Harmonic spinors, Advances in Math. 14 (1974) 1-55.

[8] H. P. McKean \& I. M. Singer, Curvature and the eigenvalues of the Laplacian, J. Differential Geometry 1 (1967) 43-69.

[9] J. Milnor, Spin Structures on manifolds, Enseignement Math. 9 (1963) 198-203.

[10] R. Parthasarathy, Dirac-Operator and discrete series, Ann. of Math. 96 (1972) 1-30.

[11] T. Sakai, On eigenvalues of Laplacian and curvature of Riemannian manifolds, Tôhoku Math. J. 23 (1971) 589-603.

[12] R. T. Seeley, Complex powers of an elliptic operator, Proc. Symp. Pure Math. Vol. 10, Amer. Math. Soc. 1967, 288-307.

[13] R. Sulankel \& R. Wintgen, Differentialgeometrie und Faserbündel, Berlin, 1972.

[14] J. A. Wolf, Essential self adjointnes for the Dirac-operator and its square, Indiana Univ. Math. J. 22 (1973) 611-640.

[15] _ Spaces of constant curvature, McGraw-Hill, New York, 1967.

HUMBOLDT-UNIVERSITÄT ZU BERLIN 